\title{
Pyogenic liver abscess as a complication of sigmoid polypectomy
}

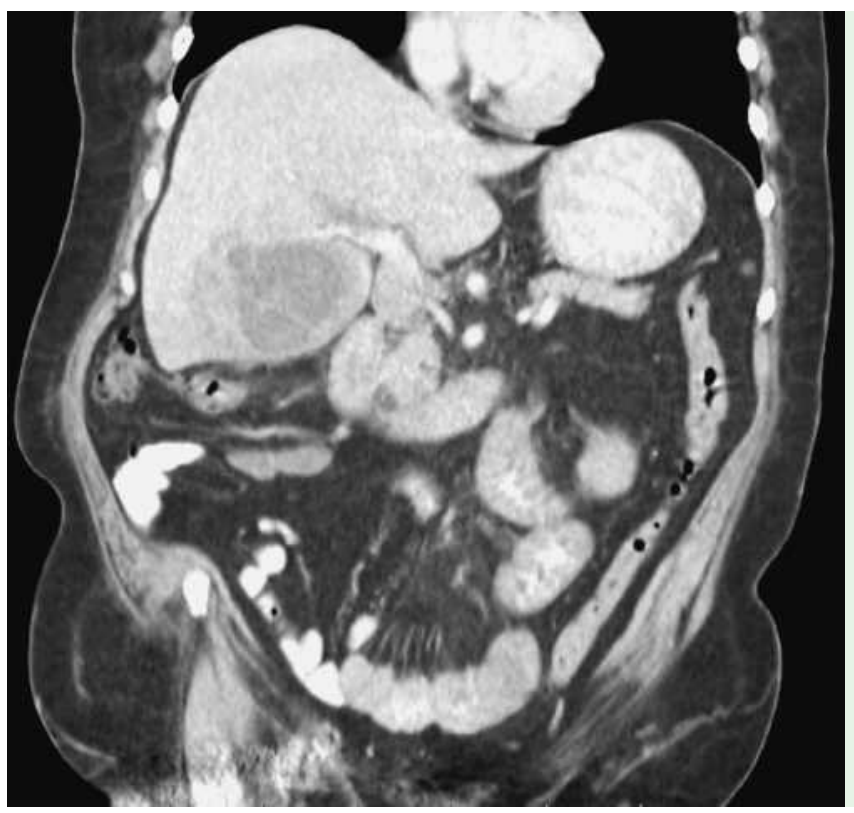

Fig. 1 Computed tomographic view (coronal reconstruction) showing the pyogenic liver abscess in the right lobe of the liver.

A previously well 65-year-old man underwent a routine colonoscopy after being referred with diarrhea. A $15-\mathrm{mm}$ pedunculated polyp was observed at the rectosigmoid junction and an uncomplicated polypectomy was performed; examination to the cecum was otherwise normal. He was discharged 3 hours after this procedure. Histological examination of the polyp revealed a tubulovillous adenoma with low-grade dysplasia. The patient returned 3 days later with fevers and rigors, but with no abdominal pain. The symptoms had begun 12 hours after the colonoscopy, and on admission he was found to be hypotensive and septic, with a lowgrade fever. Clinical examination was unremarkable. He had a neutrophil leukocytosis of $43.6 \times 10^{9} / \mathrm{L}$ and a C-reactive protein level of $218 \mathrm{mg} / \mathrm{L}$, but liver function tests were normal. Blood cultures grew Streptococcus milleri, and appropriate antibiotic therapy was commenced. Despite this treatment, however, he continued to show signs of sepsis. Abdominal computed tomography 6 days after his colonoscopy demonstrated a multiloculated, right-lobe liver abscess ( $\bullet$ Fig. 1), and $300 \mathrm{~mL}$ of purulent fluid was aspirated from this, which also grew $S$. milleri. After drainage of this abscess he made an uncomplicated recovery.
We believe that he developed the pyogenic liver abscess after the polypectomy. The likely pathophysiological mechanism was the development of a pylephlebitis following the polypectomy, with subsequent seeding to the liver. The abdominal computed tomographic scan was performed 6 days after the polypectomy, which was enough time for a large abscess to form. Pylephlebitis is a recognized complication of intra-abdominal infection and colonic perforation [1]. Indeed, pyogenic liver abscesses have been described as a presenting feature of colonic tubulovillous adenoma [2]. S. milleri is a common cause of liver abscess and this has been described as a complication following the colonscopic removal of an impacted fish bone [3]. We believe this to be first case report of a $S$. milleri liver abscess complicating polypectomy of a tubulovillous adenoma in the sigmoid colon.

Endoscopy_UCTN_Code_CPL_1AJ_2AC

\section{A. D. Farmer ${ }^{1}$, K. Browett ${ }^{1}$, V. Rusius ${ }^{1}$, S. Bhalerao ${ }^{2}$, M. R. Anderson ${ }^{1}$ \\ 1 Department of Gastroenterology, City Hospital, Birmingham, United Kingdom \\ 2 Department of Surgery, City Hospital, Birmingham, United Kingdom}

\section{References}

1 Saxena R, Adolph M, Ziegler JR et al. Pylephlebitis: a case report and review of outcome in the antibiotic era. Am J Gastroenterol 1996; 91: $1251-1253$

2 Lai HC, Chan CY, Peng CYet al. Pyogenic liver abscess associated with large colonic tubulovillous adenoma. World J Gastroenterol 2006; 12: 990-992

3 Paraskeva KD, Bury RW, Isaacs P. Streptococcus milleri liver abscesses: an unusual complication after colonoscopic removal of an impacted fish bone. Gastrointest Endosc 2000; 51: 357-358

Bibliography

DOI 10.1055/s-2007-966549

Endoscopy 2007; 39: 261

(c) Georg Thieme Verlag KG Stuttgart · New York . ISSN 0013-726X

Corresponding author

\section{A. D. Farmer}

Department of Gastroenterology

Sandwell and West Birmingham NHS Trust

City Hospital

Dudley Road

Birmingham B18 7QH

United Kingdom

Fax: +44-1785253672

penv@mac.com 